\title{
Genome Sequence of the Biocontrol Agent Coniothyrium minitans Conio (IMI 134523)
}

\author{
Denise Patel, ${ }^{1}$ Taiwo A. Shittu, ${ }^{1}$ Riccardo Baroncelli, ${ }^{1,2}$ Sreenivasaprasad Muthumeenakshi, ${ }^{1}$ \\ Thomas H. Osborne, ${ }^{1}$ Thamarai K. Janganan, ${ }^{1}$ and Surapareddy Sreenivasaprasad ${ }^{1, \dagger}$ \\ ${ }^{1}$ School of Life Sciences, Institute of Biomedical and Environmental Science and Technology (iBEST), \\ University of Bedfordshire, Luton, United Kingdom \\ ${ }^{2}$ Instituto Hispano-Luso de Investigaciones Agrarias (CIALE), Universidad de Salamanca, Villamayor, \\ Salamanca, Spain
}

\begin{abstract}
Coniothyrium minitans (synonym, Paraphaeosphaeria minitans) is a highly specific mycoparasite of the wide host range crop pathogen Sclerotinia sclerotiorum. The capability of C. minitans to destroy the sclerotia of $S$. sclerotiorum has been well recognized and it is available as a widely used biocontrol product Contans WG. We present the draft genome sequence of $C$. minitans Conio (IMI 134523), which has previously been used in extensive studies that formed part of a registration package of the commercial product. This work provides a distinctive resource for further research into the molecular basis of mycoparasitism to harness the biocontrol potential of $C$. minitans.
\end{abstract}

\section{Genome Announcement}

Coniothyrium minitans (synonym: Paraphaeosphaeria minitans [Verkley et al. 2004]) is an effective biocontrol agent of one of the most destructive soilborne plant pathogens, Sclerotinia sclerotiorum (Lib.) De Bary (Budge et al. 1995; Jones and Stewart 2000; Zeng et al. 2012). C. minitans is an ecologically obligate mycoparasite that is highly efficient in colonizing S. sclerotiorum sclerotia with little effect on the surrounding microbial populations (Whipps and Gerlagh 1992; Whipps et al. 2008). C. minitans can significantly reduce sclerotial inoculum and, also, inhibit the production of apothecia (Jones et al. 2004). The strain C. minitans Conio (IMI 134523) was originally isolated from S. sclerotiorum sclerotia colonizing potato stems in Scotland in the United Kingdom, and C. minitans has been used extensively to control sclerotinia diseases of several vegetable crops (Ashraf and Zuhaib 2013; Sun et al. 2017).

C. minitans Conio (IMl 134523) was grown in potato dextrose broth for 10 days at $20^{\circ} \mathrm{C}$. The mycelium was harvested using sterile filter paper, was air dried at room temperature for $15 \mathrm{~min}$, and was ground into a fine powder with liquid nitrogen, using a sterile pestle and mortar. The genomic DNA was extracted from the mycelial powder using a modified CTAB method (Shittu 2018). The genome sequence of $C$. minitans Conio was generated using a combination of Illumina MiSeq-600 and PacBio-Sequel platforms, utilizing the services provided by the University of Cambridge, United Kingdom, and McGill University, Quebec, Canada. For Illumina sequencing, the paired-end (PE) and mate pair (MP) libraries were

\footnotetext{
${ }^{\dagger}$ Corresponding author: S. Sreenivasaprasad; ss.prasad@beds.ac.uk
}

The author(s) declare no conflict of interest.

Accepted for publication 28 October 2020.

Copyright ( $) 2021$ The Author(s). This is an open access article distributed under the CC BY-NC-ND 4.0 International license.
Funding

This research is part of a project funded by The Richard Ormonde Shuttleworth Remembrance Trust, Bedfordshire, SG18 9EP, U.K. Project BIOIN-SARET.

\section{Keywords}

biocontrol, Coniothyrium minitans, epipolythiodioxopiperazine, microbial ecology, mycoparasite, Sclerotinia sclerotiorum, secondary metabolism 
Table 1. Genome assembly statistics of Coniothyrium minitans Conio (IMI 134523)

\begin{tabular}{lc} 
Features & Value \\
Number of scaffolds & 34 \\
Genome assembly size (bp) & $47,922,910$ \\
Largest scaffold (bp) & $5,154,056$ \\
N50 (bp) & $2,642,187$ \\
L50 & 7 \\
BUSCO completeness (\%) & 97.7 \\
Total number of predicted genes & 13,677 \\
\hline
\end{tabular}

prepared with the TruSeq PCR-free and Nextera MP kits, using $550 \mathrm{bp}$ and 2.5 to $4 \mathrm{~kb}$ DNA fragments, respectively. For the ultralong read PacBio sequencing, a single molecule realtime (SMRT) bell library of 20-kb fragments was prepared. A total of approximately 16.53 million reads were generated from the PE $(4,493,194)$, MP $(11,458,494)$, and SMRT bell $(593,557)$ libraries, together yielding approximately 12.38 billion bases. FastQC v0.11.5 was used to assess the quality of the Illumina reads. Low-quality bases, with a Phred score of less than Q20, and adaptor sequences, where present, were filtered using Trimmomatic v0.36 (Bolger et al. 2014) in the PE reads and BBDuk plugin within Geneious v9.1.5 in the MP reads (Kearse et al. 2012). Curated Illumina reads (PE and MP) comprising approximately 3.92 billion bases and PacBio reads comprising approximately 7.78 billion bases were assembled using SPAdes v3.5.0 (Bankevich et al. 2012) and CANU v1.6 (Koren et al. 2017), respectively. The assembled genome from the PacBio reads was polished with the high-quality reads from Illumina PE sequencing, using the program Pilon (Walker et al. 2014). The polished assembly was then integrated into the genome assembled from the lllumina reads using the Quickmerge program (Chakraborty et al. 2016).

The nuclear genome of $C$. minitans Conio was assembled into 34 scaffolds, with a total assembly size of $47.9 \mathrm{Mb}$ (48.06\% GC content), based on an approximate coverage of 226x. BUSCO v4.0.0 analysis (Waterhouse et al. 2018) utilizing the Ascomycota_odb10 set (1,706 genes) revealed $97.7 \%$ completeness of the genome assembly and additional genome statistics are provided in Table 1. The gene set in the nuclear genome was predicted using MAKER3 v3.01.02 (Baroncelli et al. 2016; Holt and Yandell 2011). The ab initio gene predictor Augustus was trained using RNAseq data of $C$. minitans ZS-1, available in GenBank (Zhao et al. 2020). Overall, 13,677 protein-coding gene models were predicted. Using antiSMASH v5.12 (Medema et al. 2011), 15 putative secondary metabolite gene clusters were identified, including those encoding nonribosomal peptides, polyketides, and terpenoids. A 30-kb region within scaffold 10 showed similarity to gene clusters encoding epipolythiodioxopiperazine (ETP)-type secondary metabolites known to have diverse biological roles (Muthumeenakshi et al. 2007; Vargas et al. 2014). Of the 15 clusters, seven showed no similarity to any known secondary metabolite gene clusters in the database and therefore could be specific to C. minitans Conio. At present, only one C. minitans genome sequence (isolate ZS-1) is available in the public domain (Zhao et al. 2020) and an initial comparison of the genome sequence of Conio has suggested a large structural variation in terms of the genome size and gene content.

Despite the successful development of $C$. minitans as a biocontrol agent for commercial application, various biological and environmental factors are known to affect its efficacy and consistency (Nicot et al. 2019; Whipps et al. 2008; Zhao et al. 2020). Abiotic factors such as temperature, $\mathrm{pH}$, light, and water potential are known to affect key biological attributes such as spore germination, hyphal extension, and pycnidial production in C. minitans isolates. This can impact the efficacy of $C$. minitans to successfully colonize and degrade the sclerotia of the host S. sclerotiorum (Jones et al. 2011; McQuilken et al. 1997). Qualitative or quantitative differences, or both, in secondary metabolite production exist in C. minitans. For example, strain Conio is known to produce macrosphelide $\mathrm{A}$, which inhibits $\mathrm{S}$. sclerotiorum and various other microbes (McQuilken et al. 2003). However, among 13 C. minitans isolates representing a worldwide collection, the inhibitory effect varied considerably (Tomprefa et al. 2009). Notable variation in the mycoparasitic ability of $C$. minitans was observed among 39 isolates from different geographic locations and strain Conio from the United Kingdom was ranked as 
a high-performing isolate (Jones and Stewart 2000). Among 75 S. sclerotiorum isolates from different regions of France, wide differences were reported in their susceptibility to a monoconidial isolate from Contans (Nicot et al. 2019). Genes such as CmpacC, encoding a transcription factor (Lou et al. 2015), and Cmoxdc1, encoding oxalate decarboxylase (Ren et al. 2010), have been shown to regulate key functions associated with the mycoparasitic processes of $C$. minitans. The ETP-type gene cluster in $C$. minitans Conio differs in gene number, order, and orientation from the corresponding gene clusters in Aspergillus and Trichoderma species but contains the core gene CmgliP, involved in gliotoxin biosynthesis (Muthumeenakshi et al. 2007). Disruption of this gene in Trichoderma virens halted the production of gliotoxin and significantly reduced the mycoparasitic ability (Vargas et al. 2014). Along with Conio, the genome sequences of a collection of $C$. minitans isolates representing diverse geographic locations have been generated, which would enable further analysis of the ETP-type gene cluster and one or more of the putative metabolites.

The genome sequence of strain Conio (IMI 134523) represents a distinctive resource for further research into the mechanisms underpinning the ecological competence of $C$. minitans as a biocontrol agent, including the discovery of novel genes and their products. Investigations integrating comparative and functional genomics with appropriate biological and pathological experimentation offer the scope to identify genome-wide similarities and differences in $C$. minitans related to differing levels of mycoparasitic and biocontrol capabilities. This will also assist in gaining new insights new insights into the associated sensing, signaling, and gene regulation processes. Improved molecular level understanding of how C. minitans works as a biocontrol agent and the various factors that influence its efficacy would pave the way to develop refined formulations and effective disease management strategies. The genome sequence of strain Conio (IMI 134523) has been deposited in GenBank under the accession number WJXW00000000 (BioProject PRJNA588476, BioSample SAMN13245827) and the version described in this paper is WJXW01000000.

\section{Literature Cited}

Ashraf, S., and Zuhaib, M. 2013. Fungal biodiversity: A potential tool in plant disease management. Springer, Dordrecht, The Netherlands.

Bankevich, A., Nurk, S., Antipov, D., Gurevich, A. A., Dvorkin, M., Kulikov, A. S., Lesin, V. M., Nikolenko, S. I., Pham, S., Prjibelski, A. D., Pyshkin, A. V., Sirotkin, A. V., Vyahhi, N., Tesler, G., Alekseyev, M. A., and Pevzner, P. A. 2012. SPAdes: A new genome assembly algorithm and its applications to single-cell sequencing. J. Comput. Biol. 19:455-477.

Baroncelli, R., Amby, D. B., Zapparata, A., Sarrocco, S., Vannacci, G., Le Floch, G., Harrison, R. J., Holub, E., Sukno, S. A., Sreenivasaprasad, S., and Thon, M. R. 2016. Gene family expansions and contractions are associated with host range in plant pathogens of the genus Colletotrichum. BMC Genomics 17:555.

Bolger, A. M., Lohse, M., and Usadel, B. 2014. Trimmomatic: A flexible trimmer for Illumina sequence data. Bioinformatics 30:2114-2120.

Budge, S. P., McQuilken, M. P., Fenlon, J. S., and Whipps, J. M. 1995. Use of Coniothyrium minitans and Gliocladium virens for biological control of Sclerotinia sclerotiorum in glasshouse lettuce. Biol. Control 5:513-522.

Chakraborty, M., Baldwin-Brown, J. G., Long, A. D., and Emerson, J. J. 2016. Contiguous and accurate de novo assembly of metazoan genomes with modest long read coverage. Nucleic Acids Res. 44:e147.

Holt, C., and Yandell, M. 2011. MAKER2: An annotation pipeline and genomedatabase management tool for second-generation genome projects. BMC Bioinformatics 12:491.

Jones, E. E., Clarkson, J. P., Mead, A., and Whipps, J. M. 2004. Effect of inoculum type and timing of application of Coniothyrium minitans on Sclerotinia sclerotiorum: Influence on apothecial production. Plant Pathol. 53:621-628.

Jones, E. E., and Stewart, A. 2000. Selection of mycoparasites of sclerotia of Sclerotinia sclerotiorum isolated from New Zealand soils. N. Z. J. Crop Hortic. Sci. 28:105-114.

Jones, E. E., Stewart, A., and Whipps, J. M. 2011. Water potential affects Coniothyrium minitans growth, germination and parasitism of Sclerotinia sclerotiorum sclerotia. Fungal Biol. 115:871-881.

Kearse, M., Moir, R., Wilson, A., Stones-Havas, S., Cheung, M., Sturrock, S., Buxton, S., Cooper, A., Markowitz, S., Duran, C., Thierer, T., Ashton, B., Meintjes, P., and
Drummond, A. 2012. Geneious Basic: An integrated and extendable desktop software platform for the organization and analysis of sequence data. Bioinformatics 28:1647-1649.

Koren, S., Walenz, B. P., Berlin, K., Miller, J. R., Bergman, N. H., and Phillippy, A. M. 2017. Canu: Scalable and accurate long-read assembly via adaptive $k$-mer weighting and repeat separation. Genome Res. 27:722-736.

Lou, Y., Han, Y., Yang, L., Wu, M., Zhang, J., Cheng, J., Wang, M., Jiang, D., Chen, W., and Li, G. 2015. CmpacC regulates mycoparasitism, oxalate degradation and antifungal activity in the mycoparasitic fungus Coniothyrium minitans. Environ. Microbiol. 17:4711-4729.

McQuilken, M. P., Budge, S. P., and Whipps, J. M. 1997. Effects of culture media and environmental factors on conidial germination, pycnidial production and hyphal extension of Coniothyrium minitans. Mycol. Res. 101:11-17.

McQuilken, M. P., Gemmell, J., Hill, R. A., and Whipps, J. M. 2003. Production of macrosphelide A by the mycoparasite Coniothyrium minitans. FEMS Microbiol. Lett. 219:27-31.

Medema, M. H., Blin, K., Cimermancic, P., de Jager, V., Zakrzewski, P., Fischbach, M. A., Weber, T., Takano, E., and Breitling, R. 2011. antiSMASH: Rapid identification, annotation and analysis of secondary metabolite biosynthesis gene clusters in bacterial and fungal genome sequences. Nucleic Acids Res. 39 (suppl_2):W339-W346.

Muthumeenakshi, S., Sreenivasaprasad, S., Rogers, C. W., Challen, M. P., and Whipps, J. M. 2007. Analysis of cDNA transcripts from Coniothyrium minitans reveals a diverse array of genes involved in key processes during sclerotial mycoparasitism. Fungal Genet. Biol. 44:1262-1284.

Nicot, P. C., Avril, F., Duffaud, M., Leyronas, C., Troulet, C., Villeneuve, F., and Bardin, M. 2019. Differential susceptibility to the mycoparasite Paraphaeosphaeria minitans among Sclerotinia sclerotiorum isolates. Trop. Plant Pathol. 44:82-93.

Ren, L., Li, G., and Jiang, D. 2010. Characterization of some culture factors affecting oxalate degradation by the mycoparasite Coniothyrium minitans. J. Appl. Microbiol. 108:173-180.

Shittu, T. A. 2018. Population analysis of the finger millet blast pathogen Magnaporthe oryzae in Eastern Africa. Ph.D. thesis, University of Bedfordshire, U.K. 
Sun, X., Zhao, Y., Jia, J., Xie, J., Cheng, J., Liu, H., Jiang, D., and Fu, Y. 2017. Uninterrupted expression of CmSIT1 in a sclerotial parasite Coniothyrium minitans leads to reduced growth and enhanced antifungal ability. Front. Microbiol. 8:2208.

Tomprefa, N., McQuilken, M. P., Hill, R. A., and Whipps, J. M. 2009. Antimicrobial activity of Coniothyrium minitans and its macrolide antibiotic macrosphelide $A$. J. Appl. Microbiol. 106:2048-2056.

Vargas, W. A., Mukherjee, P. K., Laughlin, D., Wiest, A., Moran-Diez, M. E., and Kenerley, C. M. 2014. Role of gliotoxin in the symbiotic and pathogenic interactions of Trichoderma virens. Microbiology 160:2319-2330.

Verkley, G., Silva, M., Wicklow, D. T., and Crous, P. 2004. Paraconiothyrium, a new genus to accommodate the mycoparasite Coniothyrium minitans, anamorphs of Paraphaeosphaeria, and four new species. Stud. Mycol. 50:323-335.

Walker, B. J., Abeel, T., Shea, T., Priest, M., Abouelliel, A., Sakthikumar, S., Cuomo, C. A., Zeng, Q., Wortman, J., Young, S. K., and Earl, A. M. 2014. Pilon: An integrated tool for comprehensive microbial variant detection and genome assembly improvement. PLoS One 9:e112963.
Waterhouse, R. M., Seppey, M., Simão, F. A., Manni, M., Ioannidis, P., Klioutchnikov, G., Kriventseva, E. V., and Zdobnov, E. M. 2018. BUSCO applications from quality assessments to gene prediction and phylogenomics. Mol. Biol. Evol. 35: 543-548.

Whipps, J. M., and Gerlagh, M. 1992. Biology of Coniothyrium minitans and its potential use in disease biocontrol. Mycol. Res. 96:897-907.

Whipps, J. M., Sreenivasaprasad, S., Muthumeenakshi, S., Rogers, C., and Challen, M. 2008. Use of Coniothyrium minitans as a biocontrol agent and some molecular aspects of sclerotial mycoparasitism. Eur. J. Plant Pathol. 121:323-330

Zeng, W., Wang, D., Kirk, W., and Hao, J. 2012. Use of Coniothyrium minitans and other microorganisms for reducing Sclerotinia sclerotiorum. Biol. Control 60: 225-232.

Zhao, H., Zhou, T., Xie, J., Cheng, J., Chen, T., Jiang, D., and Fu, Y. 2020. Mycoparasitism illuminated by genome and transcriptome sequencing of Coniothyrium minitans, an important biocontrol fungus of the plant pathogen Sclerotinia sclerotiorum. Microb. Genom. 6:e000345. 